\title{
Experimental Determination of the Absorption Cross-Section and Molar Extinction Coefficient of Colloidal CdSe Nanoplatelets
}

\author{
Aydan Yeltik, ${ }^{\dagger}$ Savas Delikanli, ${ }^{\dagger}$ Murat Olutas, $^{\dagger, \dagger}$ Yusuf Kelestemur, $^{\dagger}$ Burak Guzelturk, ${ }^{\dagger, \S}$ \\ and Hilmi Volkan Demir ${ }^{*}$,,
}

\begin{abstract}
${ }^{\dagger}$ Department of Physics, and Department of Electrical and Electronics Engineering, UNAM-Institute of Materials Science and Nanotechnology, Bilkent University, Ankara 06800, Turkey

${ }^{\ddagger}$ Department of Physics, Abant Izzet Baysal University, Bolu 14280, Turkey

${ }^{\S}$ Luminous! Center of Excellence for Semiconductor Lighting and Displays, School of Electrical and Electronic Engineering, School of Physical and Materials Sciences, Nanyang Technological University, Singapore 639798, Singapore
\end{abstract}

Supporting Information

ABSTRACT: There has been a strong interest in solutionprocessed two-dimensional nanomaterials because of their great potential in optoelectronics. Here, the absorption crosssection and molar extinction coefficient of four and five monolayer thick colloidal CdSe nanoplatelets (NPLs) having various lateral sizes are reported. The absorption cross-section of these NPLs and their corresponding molar extinction coefficients are found to strongly depend on the lateral area. An excellent agreement is observed between the experimental results and the calculated values based on the small-particle light absorption model. With these optical properties, NPLs hold great promise for optoelectronic applications.

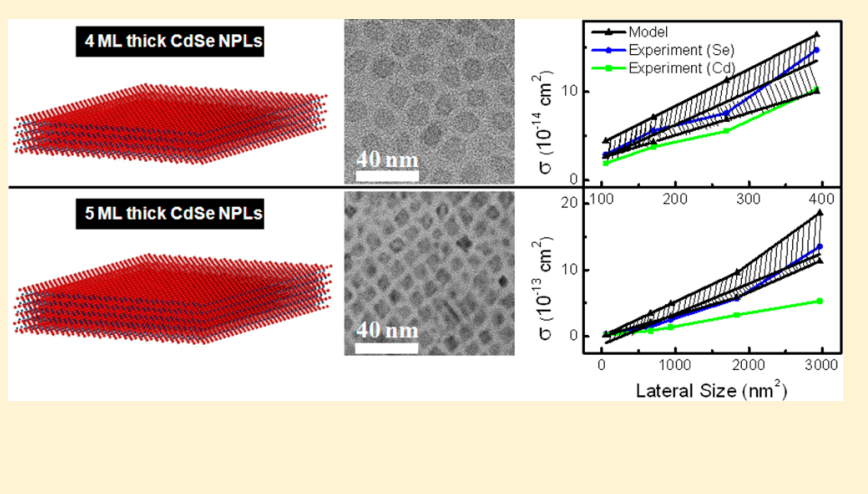

inside an NPL. Low amplified spontaneous emission threshold of the NPLs is also well explained by their large absorption cross-section. $^{16}$

Also, knowing molar extinction coefficient $(\varepsilon)$ of the NPLs is also of great importance for convenient and accurate measurements of the NPL concentrations. ${ }^{17}$ With the well-known molar extinction coefficients, different architectures of core/ shell (vertically grown shell) and core/crown (laterally grown shell) NPLs can be synthesized with great control over their thicknesses and lateral sizes.

Previous reports of individual NPL absorption cross sections indicate that NPLs exhibit higher $\sigma$ values compared to their spherical counterparts with the same size. ${ }^{16,18}$ In the work by She et al., $\sigma$ of CdSe NPL was observed to increase after the CdS shell growth, which was discussed as one of the indications of lower amplified spontaneous emission threshold compared to the core CdSe NPLs. Fluorescent lifetimes measured for CdSe NPLs were provided by Tessier et al. and much shorter values were reported compared to the ones measured for spherical QDs. ${ }^{18-20}$ In addition, Naeem et al. studied the giant oscillator strength of CdSe NPLs and concluded that decrease in the radiative lifetime is associated with the exciton coherence area, which is larger than that of spherical CdSe nanocrystals. ${ }^{21}$ Furthermore, radiative lifetimes on the order of picoseconds for

Received: September 23, 2015

Published: November 6, 2015 
NPLs were measured and/or predicted by several groups. ${ }^{2,10,21}$ In the previous literature, a complete and systematic study on the absorption cross-section of CdSe NPLs has, however, not been reported to date. There is thus a strong need for the experimental determination of the absorption cross-section of the NPLs of a specific vertical thickness as a function of their lateral size. This is particularly essential as a series of reference measurements for finding molar extinction coefficients.

In this work, we experimentally determined the absorption cross sections and molar extinction coefficients of four and five monolayer (ML) thick CdSe NPLs. For this purpose, two independent experimental methods, inductively coupled plasma optical emission spectroscopy (ICP-OES) and inductively coupled plasma mass spectroscopy (ICP-MS), were used. For both of the methods, the NPL samples were purified by efficient purification procedures. Concentrations of the NPLs were first determined via ICP-OES measurements. Then, results of the first method were confirmed by the second method, ICP-MS. In the ICP-MS measurements, concentrations were diluted to reach reliable ranges. Using these concentrations, $\sigma$ values were found with the help of absorption spectroscopy technique. Results indicate that the absorption cross sections of both the 4 and $5 \mathrm{ML}$ thick NPLs are in direct correlation with the lateral sizes. Furthermore, the experimental $\sigma$ values agree well with the calculated ones based on the Ricard model. ${ }^{22}$ The corresponding $\varepsilon$ values were also calculated using the concentrations obtained from ICP-OES, and strong lateral size dependence was observed for both NPL sets.

\section{EXPERIMENTAL SECTION}

Chemicals. Cadmium acetate dihydrate $\left(\mathrm{Cd}(\mathrm{OAc})_{2} \cdot 2 \mathrm{H}_{2} \mathrm{O}\right)$ (>98\%), selenium ( $\mathrm{Se}$ ) (99.999\% trace metals basis), technical grade 1-octadecene (ODE), and technical grade oleic acid (OA) (90\%) were purchased from Sigma-Aldrich. Cadmium (Cd) myristate was prepared at our laboratory. Hexane, methanol, and acetone were purchased from Merck Millipore.

Preparation of Cadmium Myristate. Cadmium myristate was prepared by following the procedure given in the literature. ${ }^{23}$ Concisely, $1.23 \mathrm{~g}$ of cadmium nitrate tetrahydrate was dissolved in $40 \mathrm{~mL}$ of methanol, and $3.13 \mathrm{~g}$ of sodium myristate was dissolved in $250 \mathrm{~mL}$ of methanol. The mixture was completely dissolved for complete dissolution and then gently mixed. The solution was stirred strongly for $1 \mathrm{~h}$. Following that, the solution was centrifuged, and the precipitation was dissolved in methanol. This washing step with methanol was performed at least three times to remove the excess precursors. At the end, the final precipitated part was kept under vacuum for $24 \mathrm{~h}$ for complete drying.

Synthesis of 4 ML Thick CdSe NPLs. Four monolayers of CdSe NPLs was synthesized according to the given recipe in the literature. ${ }^{23}$ First, $170 \mathrm{mg}$ of cadmium myristate, $12 \mathrm{mg}$ of Se, and $15 \mathrm{~mL}$ of ODE were loaded into a three-neck flask. Then, the solution was degassed at room temperature for half an hour to remove excess oxygen and volatile solvents. Subsequently, the solution was heated to $240{ }^{\circ} \mathrm{C}$ for $4 \mathrm{ML}$ thick NPLs under argon atmosphere. When the temperature reaches $195{ }^{\circ} \mathrm{C}, 80 \mathrm{mg}$ of cadmium acetate dehydrate was introduced swiftly into the reaction. After the growth of CdSe NPLs at $240{ }^{\circ} \mathrm{C}$ for $10 \mathrm{~min}, 0.5 \mathrm{~mL}$ of OA was injected, and the temperature of the solution was decreased to room temperature. In order to obtain these CdSe NPLs in different lateral sizes, the growth time was carefully changed. The resulting 4 ML thick CdSe NPLs synthesized with this recipe exhibit a peak emission at $513 \mathrm{~nm}$ and other side products including NPLs having different thicknesses can be removed by sizeselective precipitation. Finally, the CdSe NPLs were dissolved and stored in hexane.

Synthesis of $\mathbf{5}$ ML Thick Core CdSe NPLs. Five monolayers of CdSe NPLs was synthesized following a recipe in the literature. ${ }^{23}$ Cadmium myristate $(170 \mathrm{mg})$ and $14 \mathrm{~mL}$ of ODE were loaded into a three-neck flask and degassed for $1 \mathrm{~h}$ at room temperature. Then, the temperature was raised to 250 ${ }^{\circ} \mathrm{C}$ under argon flow, and a solution of $12 \mathrm{mg}$ Se dissolved in 1 $\mathrm{mL}$ of ODE was quickly injected. Cadmium acetate dehydrate $(120 \mathrm{mg})$ was added $1 \mathrm{~min}$ later. The solution was kept at 250 ${ }^{\circ} \mathrm{C}$ for $10 \mathrm{~min}$, and $0.5 \mathrm{~mL}$ of $\mathrm{OA}$ was injected before cooling down to room temperature. The resulting CdSe NPLs were precipitated with the addition of acetone and dispersed in hexane.

Lateral Growth of $\mathbf{5}$ ML Thick CdSe NPLs. Five monolayers of CdSe NPLs was dissolved in hexane, and 15 $\mathrm{mL}$ of ODE was loaded into a three-neck flask. The solution was degassed at room temperature for $10 \mathrm{~min}$, and the temperature was raised up to $80{ }^{\circ} \mathrm{C}$ under vacuum. The solution was kept at $80^{\circ} \mathrm{C}$ about $1 \mathrm{~h}$ for the complete removal of hexane. Then, the temperature was increased to $255{ }^{\circ} \mathrm{C}$ under argon flow. At $255{ }^{\circ} \mathrm{C}$, anisotropic growth mixture for the lateral extension of CdSe NPLs was injected at a rate of 3 $\mathrm{mL} / \mathrm{h}$. The lateral size of the CdSe NPLs can be tuned by adjusting the injection amount. After the injection, the temperature was quickly cooled down to room temperature. The resulting $5 \mathrm{ML}$ of CdSe NPLs was precipitated with the addition of acetone and dispersed in hexane.

Extraction and Precipitation Procedures. Unreacted Cd precursors were removed by using methanol since the NPLs are insoluble in this solution while the $\mathrm{Cd}$ precursors are highly soluble. Excess amount of methanol was added into NPLs dispersed in hexane to dissolve $\mathrm{Cd}$ precursors in methanol. Then, the mixture was centrifuged and dissolved in hexane. This process was repeated three times for the removal of excess Cd precursors. The final product was redissolved in hexane for the following measurements.

ICP-OES and ICP-MS Measurements. A solution of $2 \mathrm{~mL}$ of purified $4 \mathrm{ML}$ thick NPL sample and $3 \mathrm{~mL}$ of purified $5 \mathrm{ML}$ thick NPL sample was carefully dried by gentle heating in a fume hood. Then the residual was dissolved in $20 \mathrm{~mL}$ of nitric acid solution. The digested sample was directly transferred into a volumetric flask for the ICP-OES measurement. For the ICPMS measurement, the samples were diluted by 100 times to reach the $\mathrm{ppb}$ ranges necessary for the measurement. The selenium and cadmium molar concentrations $\left(C_{\mathrm{Se}}\right.$ and $\left.C_{\mathrm{Cd}}\right)$ were determined using an Agilent 725 ICP-OES system and a Thermo XSERIES II ICP-MS system. The dimensions of the CdSe NPLs were measured from transmission electron microscopy (TEM) images. The NPL concentrations $\left(C_{\mathrm{NPL}}\right)$ using the Se concentrations were calculated according to the relation $C_{\mathrm{NPL}}=C_{\mathrm{Se}(\mathrm{Cd})} V_{\text {unit }} /\left(4 V_{\mathrm{NPL}}\right)$, where $V_{\text {unit }}$ is the volume of the CdSe unit cell and $V_{\mathrm{NPL}}$ is the physical volume of CdSe NPL. To obtain the concentrations of NPLs from the Cd concentrations, we also included the extra Cd layer of the NPLs into the calculations. Herein we assumed that the NPL has the same density as its bulk material.

UV-Vis Measurements. The purified NPLs were dispersed in a suitable amount of desired solvent to obtain reliable UV-vis absorbance spectra at room temperature (Cary 100 UV-vis spectrophotometer). The absorbance values were used 
(a) Wavelength $(\mathrm{nm})$
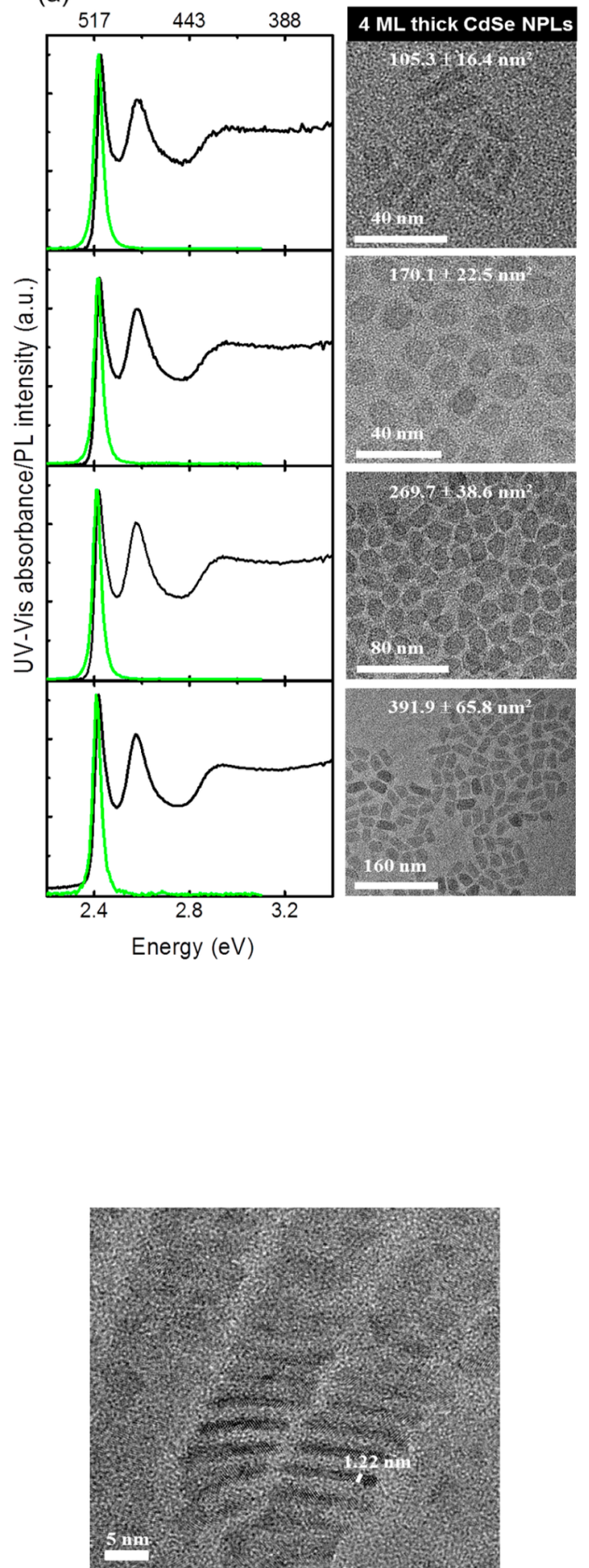

(b) Wavelength $(\mathrm{nm})$
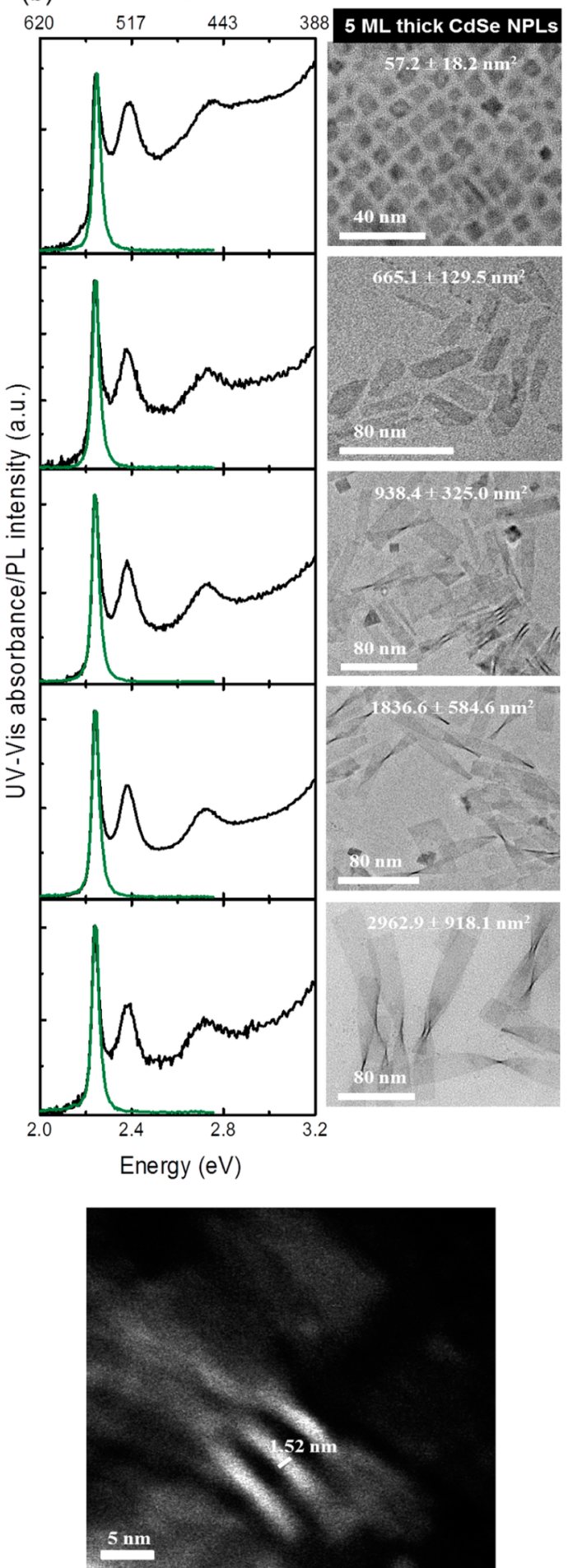

Figure 1. UV-vis absorbance spectra, PL spectra, and TEM images of the (a) $4 \mathrm{ML}$ and (b) 5 ML thick CdSe NPLs having various lateral sizes.

to calculate the absorption cross sections and the corresponding molar extinction coefficients at room temperature.

\section{RESULTS AND DISCUSSION}

Synthesis and Characterization of Nanoplatelets. The 4 and 5 ML thick CdSe NPLs used for the experiments were synthesized through the recently reported approach of Tessier et al. with a slight modification. ${ }^{23}$ Using this route, we achieved to synthesize NPLs with varying lateral sizes. Figure $1 \mathrm{a}, \mathrm{b}$ shows the TEM images and the absorption and photoluminescence (PL) spectra of 4 and 5 ML NPL samples. TEM measurements of the NPLs revealed that they are approximately square- or rectangular-shaped. We used these high quality TEM images to determine the NPL areas, and the NPL shapes were approximated to squares and rectangles, which is a standard procedure in the literature to obtain the areas. The folded NPLs were also carefully analyzed in terms of the shape and folding angle to obtain the correct areas. To this end, more than 300 
NPLs were measured to obtain each NPL area. The error bars presented in the study include also the errors originated from the folding of the large NPLs. In addition, the thicknesses are $\sim 1.22 \mathrm{~nm}$ for the 4 ML NPLs and $\sim 1.52 \mathrm{~nm}$ for the $5 \mathrm{ML}$ NPLs as seen from Figure 1a,b. Also, their QYs were measured to be $\sim 30-50 \%$ using the standard QY measurement with Rhodamine6G described elsewhere. ${ }^{24}$ This QY level is high compared to colloidal only core CdSe QDs. The emission peak positions and their full width at half-maximum (fwhm) are almost independent of the platelet area for both the 4 and 5 ML thick NPLs as observed in Figure 2a,b. For the 4 ML NPLs,
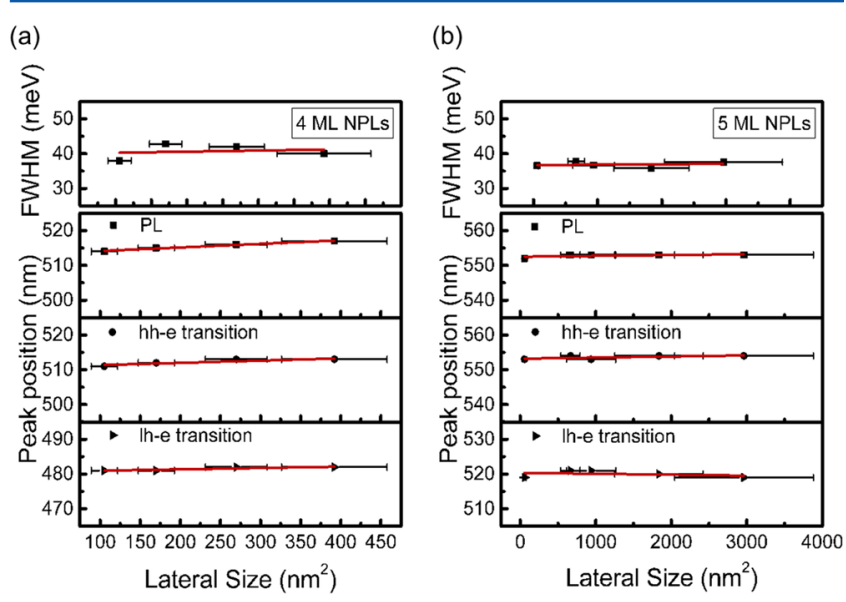

Figure 2. Peak positions of the PL fwhm, PL, hh-e transition, and lh-e transition of (a) 4 ML NPLs and (b) 5 ML NPLs for varying lateral sizes.

the emission peak is obtained at $\sim 513 \mathrm{~nm}$ with a fwhm of $\sim 8$ $\mathrm{nm}$, and it is at $\sim 553 \mathrm{~nm}$ with a fwhm of $\sim 10 \mathrm{~nm}$ for the $5 \mathrm{ML}$ NPLs. Similarly, the first and second absorption peaks are also independent of the NPL lateral area as given in Figure 2a,b. The first absorption peak is at $\sim 513 \mathrm{~nm}$ for the 4 ML NPLs and at $\sim 553 \mathrm{~nm}$ for the 5 ML NPLs. The second absorption peak is at $\sim 482$ for the 4 ML NPLs, while it is at $\sim 520 \mathrm{~nm}$ for the $5 \mathrm{ML}$ ones. These first and second absorption peaks correspond to the heavy hole-electron (hh-e) and light holeelectron (lh-e) transitions of the NPLs, respectively. ${ }^{2}$

In this work, it was critical to remove excess cadmium to the best possible extent. Therefore, the samples were subjected to the cleaning procedures described in the Extraction and Precipitation Procedures section. Conveniently, the purification was monitored by UV-vis absorbance. The absorption spectra of the host medium, the as-synthesized sample, and the sample after cleaning are shown in Figure S1 (see the Supporting Information). Further discussion for the $\mathrm{Cd} / \mathrm{Se}$ ratios is given in the following section.

Absorption Cross-Section and Corresponding Molar Extinction Coefficient of Nanoplatelets via Inductively Coupled Plasma Optical Emission Spectroscopy. We obtained the absorption cross sections of each NPL sample based on the Se and Cd concentrations. First, the NPL concentration was determined by dissolving the platelets in a nitric-acid solution and by measuring the ion concentrations using ICP-OES. Then, the NPL concentrations were calculated by extracting the dimensions from TEM images and using the relations given above in the ICP-OES and ICP-MS Measurements section. The per-particle $\sigma$ values for the 4 ML NPLs having various areas were determined by using the formula, $\sigma=$ $2303 A /\left(C_{\mathrm{NPL}} N_{\mathrm{A}} L\right)$, where $A$ is the absorbance, $N_{\mathrm{A}}$ is the Avogadro's number, and $L$ is the optical path length. ${ }^{16}$ The results are presented as a function of the absorption energy in Figure $3 a$ using the Se concentration and Figure $3 b$ using the $\mathrm{Cd}$ concentration. As given in the figure, the absorption crosssection was observed to increase with the platelet area over the whole absorbance spectrum.
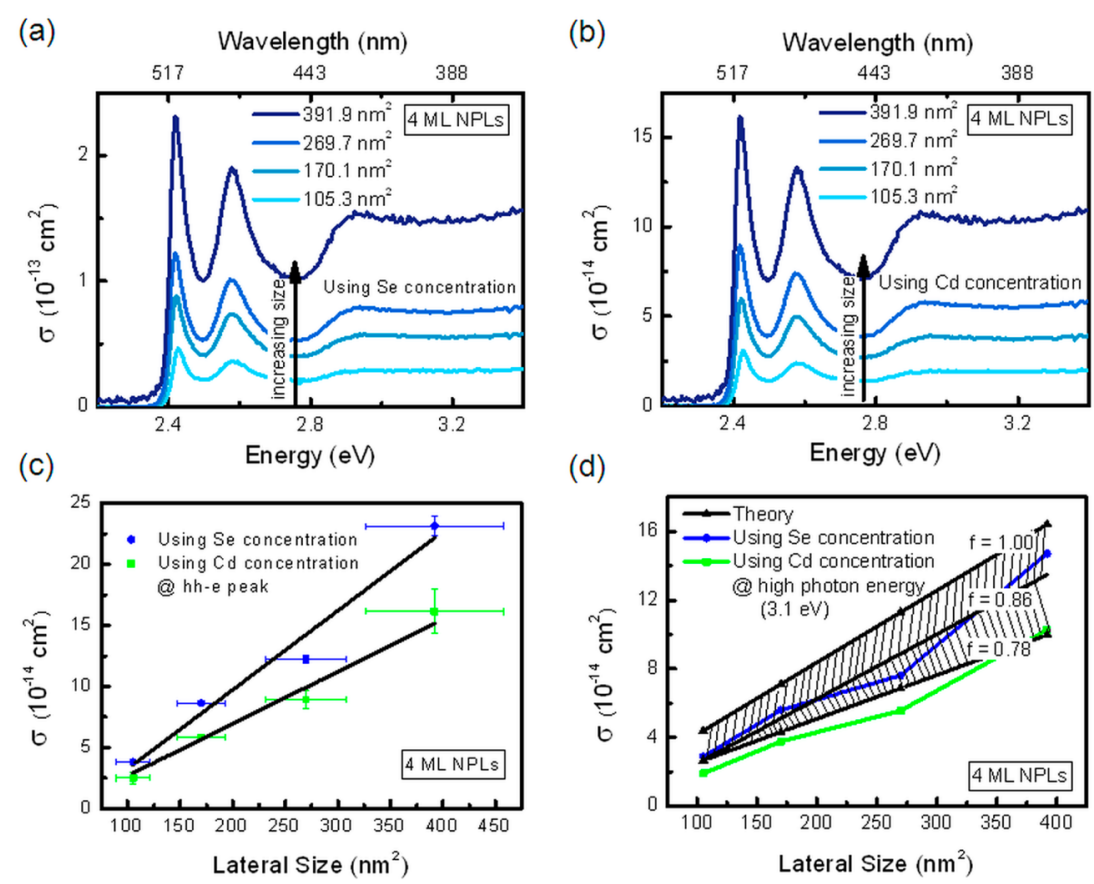

(d)

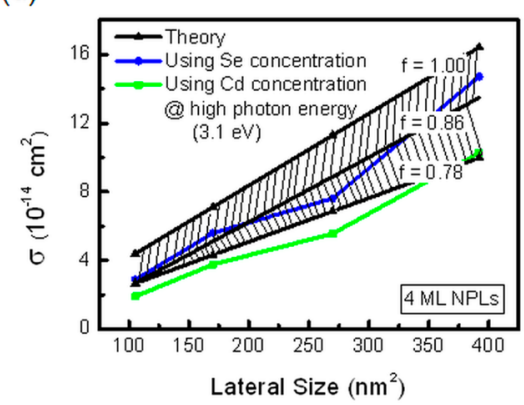

Figure 3. Absorption cross-section spectra of 4 ML CdSe NPL samples with varying lateral sizes obtained by using the (a) Se and (b) Cd concentrations from ICP-OES measurements. Lateral size dependence of the absorption cross-section (c) at the energy of hh-e transition peak and (d) at high photon energy $3.1 \mathrm{eV}$. 
(a)

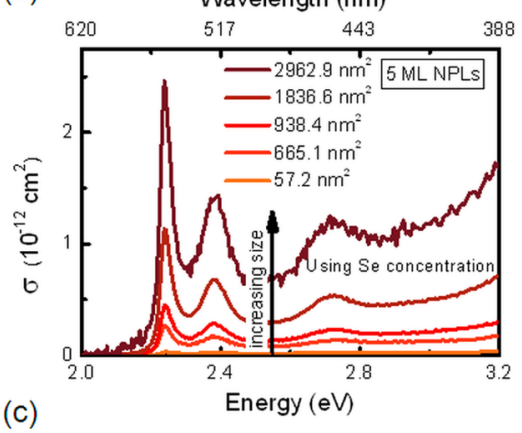

(C)

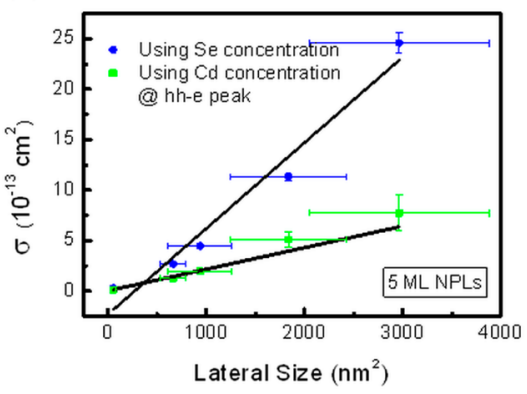

(b)

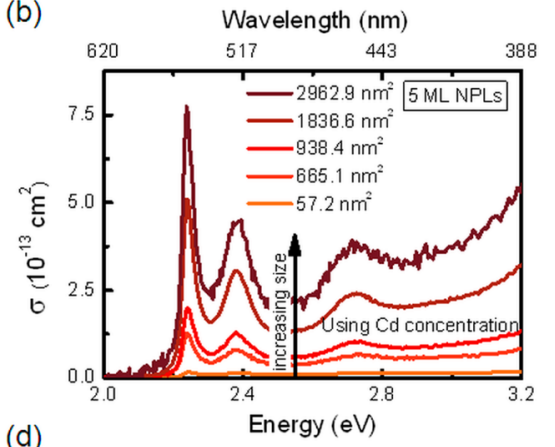

(d)

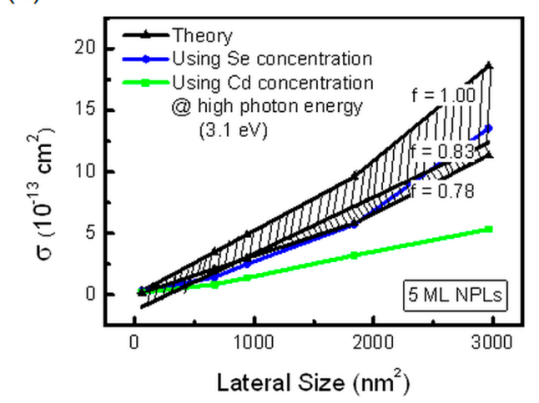

Figure 4. Absorption cross-section spectra of $5 \mathrm{ML}$ CdSe NPL samples with varying lateral sizes obtained by using the (a) Se and (b) Cd concentrations from ICP-OES measurements. Lateral size dependence of the absorption cross-section (c) at the energy of hh-e transition peak and (d) at high photon energy $3.1 \mathrm{eV}$.

The observation of lower absorption cross sections extracted using the Cd concentrations compared to those from the Se concentrations indicate a higher amount of $\mathrm{Cd}$ in the NPL samples, which is also common for colloidal QDs. To investigate this observation, the Cd-to-Se molar ratio $(\mathrm{Cd} / \mathrm{Se}$ ratio) of the purified samples was calculated by using the results of ICP-OES measurements. The ratio was found to be $1.80 \pm$ 0.07 for the $4 \mathrm{ML}$ NPL sample and $1.72 \pm 0.11$ for the $5 \mathrm{ML}$ NPL sample. The ICP-MS analysis was also performed and similar ratios were obtained as with the ICP-OES analysis (1.64 \pm 0.12 for the $4 \mathrm{ML} N P L$ sample and $1.39 \pm 0.10$ for the $5 \mathrm{ML}$ NPL sample). For the further confirmation, energy dispersive $\mathrm{X}$-ray spectroscopy experiments before and after cleaning procedures were conducted and, observing the similar results with the ICP, there existed around 1.5 times improvement on the $\mathrm{Cd} / \mathrm{Se}$ ratios after cleaning. However, all these values are still higher than the theoretical values as discussed in the study by $\mathrm{Li}$ et al. ${ }^{25}$ The reasons for these higher ratio values are mainly 2-fold: First, not only the basal planes but also the side facets of the NPLs terminate with $\mathrm{Cd}^{2+}$ ions. ${ }^{25}$ Second, the $\mathrm{Cd}$ precursor was in excess during the synthesis. Also, the cleaning process with acetone/methanol may not completely remove the excess Cd. As a result, measuring the Se content should provide a more reliable approach in determining the absorption crosssection, which will thus be used for the extraction of molar extinction coefficient.

We also investigated the lateral size dependence of the absorption cross-section for the hh-e absorption band by using the results in Figure 3a,b. The absorption cross sections (both using the Se and Cd concentrations) were observed to depend on the platelet area as can be seen in Figure 3c. The single $\sigma$ value previously reported in the study of She et al. is comparable to that of our $4 \mathrm{ML} \mathrm{NPL}$ with the similar size obtained from the Se concentration. ${ }^{16}$ In addition, the experimental $\sigma$ values for high photon energy at $3.1 \mathrm{eV}$ are shown in Figure 3d. Here those obtained from the $\mathrm{Cd}$ concentration were observed to be lower than those from the Se concentration as expected. More importantly, the $\sigma$ trend indicates only a slight nonlinearity in the dependence of the platelet area.

To understand these observations, we used a theoretical model derived by Ricard et al.: $22,26,27$

$$
\sigma(\omega)=\frac{\omega}{n_{s} c} V_{\mathrm{NPL}}|f(\omega)|^{2} \varepsilon_{2, \mathrm{NPL}}(\omega)
$$

where $n_{s}$ is the refractive index of the nanostructure's surrounding medium, $c$ is the speed of light, and $\varepsilon_{2, \mathrm{NPL}}(\omega)$ is the imaginary part of the dielectric constant at frequency $\omega$, which is obtained from the literature. ${ }^{27,28}$ The $\sigma$ values resulting from the model are also presented in Figure 3d. Here, the localfield correction factor $|f(\omega)|^{2}$ is taken in the range between $(0.78)^{2}$ and $(1.00)^{2}$, which are the corresponding values for the colloidal CdSe nanowire in hexane and the epitaxial CdSe quantum well. $^{27,29}$ Using the Se concentrations of ICP-OES measurements, the local-field correction factor for the $4 \mathrm{ML}$ NPLs from the best numerical fit to the experimental results was found as $\sim(0.86)^{2}$, which falls between the values for the colloidal nanowire and the epitaxial quantum well. This suggests that local-field correction factors of our NPLs are in the correct range. Here, the refractive index of the surrounding medium and the structure geometry are the key parameters in the calculations of local field correction factors and to reach this conclusion about the $\sigma$ values. It is also worth noting that, for the theoretical $\sigma$ calculations, we chose the high energy value, $3.1 \mathrm{eV}$, since this model is only valid in the energy region where the confinement effects are insignificant and the NPL absorption is equivalent to that of the bulk case.

The absorption cross sections of the $5 \mathrm{ML}$ NPLs were also obtained over the absorbance spectrum similar to the $4 \mathrm{ML}$ NPLs. For these measurements, the same sample preparation procedure was followed as in the ICP-OES measurements of the $4 \mathrm{ML}$ NPLs. The $\sigma$ results for the $5 \mathrm{ML}$ are shown as a 
(a)

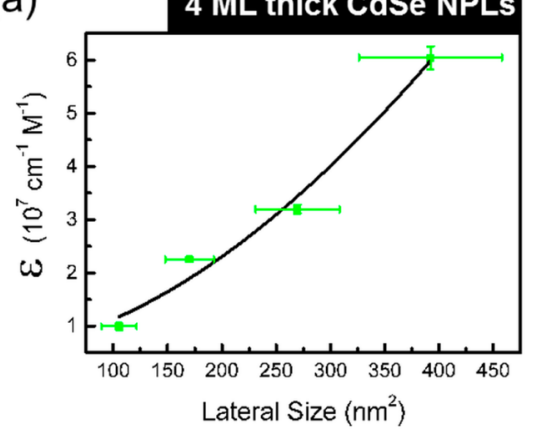

(b) 5 ML thick CdSe NPLs

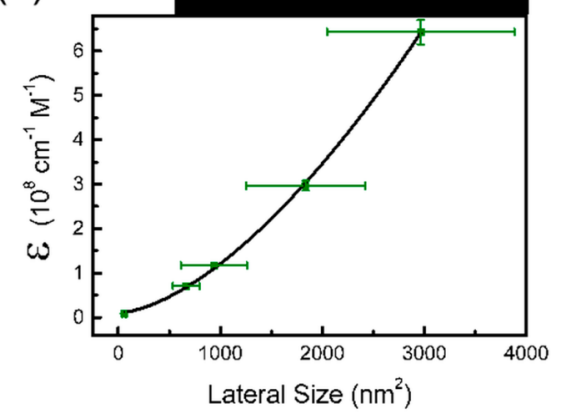

Figure 5. Lateral size dependence of the extinction coefficients of (a) 4 ML CdSe NPLs and (b) 5 ML CdSe NPLs obtained by using the Se concentrations from ICP-OES measurements.

function of the absorption energy in Figure 4a using the Se concentration and Figure $4 \mathrm{~b}$ using the $\mathrm{Cd}$ concentration. Similar to the $4 \mathrm{ML}$ NPL case, here the resulting absorption cross sections were also observed to increase with the platelet area over the whole absorbance spectrum. Again, the $\sigma$ values obtained from the $\mathrm{Cd}$ concentrations are smaller than those from the Se concentrations due to the excess of $\mathrm{Cd}$ in the samples.

For the 5 ML NPLs, the absorption cross sections for the hhe absorption band were also obtained by using the results in Figure $4 \mathrm{a}, \mathrm{b}$, and it was observed that there is a strong lateral size dependence of $\sigma$ (Figure 4c). Tessier et al. reported a similar level of absorption cross-section for a single NPL size, which is comparable to the ones presented here. ${ }^{30}$ Herein, the $\sigma$ trend of the main transition band obtained using the $\mathrm{Cd}$ ion concentration exhibits a different slope compared to that obtained using the Se ion concentration. This is probably due to the effect of the excess $\mathrm{Cd}$ in the sample, increasing with the lateral area. Moreover, the experimental absorption cross sections at high photon energy $3.1 \mathrm{eV}$ are also presented in Figure $4 d$, and the values obtained from the $\mathrm{Cd}$ concentrations are again lower than those from the Se concentrations. For the theoretical $\sigma$ calculations, we again used the Ricard model. The local-field correction factor for the $5 \mathrm{ML}$ NPLs from the best numerical fit to the experimental data using the Se concentrations of ICP-OES measurements was found to be $\sim(0.83)^{2}$, which is between the values of the colloidal nanowire and the epitaxial quantum well. This is a very similar result as in the case of the 4 ML NPL samples.

The size-dependent molar extinction coefficients at the bandedge of CdSe QDs were previously reported, and these studies demonstrated that molar extinction coefficient increases with size of CdSe QD. ${ }^{17,31,32}$ Additionally, it was presented that the molar absorption cross sections and corresponding extinction coefficients of $\mathrm{CdSe}$ nanorods and $\mathrm{CdSe}$ nanowires increase with size. ${ }^{33,34}$ In the light of these results, we extracted the extinction coefficients of 4 and $5 \mathrm{ML}$ CdSe NPLs from the absorption cross sections obtained via the ICP-OES measurements of the Se concentrations. The molar extinction coefficients at the transition energy of the hh-e transition peak are given in Figure 5a for the 4 ML NPLs and in Figure 5b for the 5 ML NPLs. As can be seen from the figures, there is a significant dependence of the extinction coefficient on the lateral area. Using the numerical fitting analysis, we obtained general relations between the molar extinction coefficient and the NPL lateral area given in eq 2 for the 4 ML NPLs and eq 3 for the 5 ML NPLs.

$$
\begin{aligned}
& \text { 4ML CdSe NPLs: } \\
& \qquad \varepsilon=6130012 \pm 2846504+1861 \pm 170(L S)^{1.72}
\end{aligned}
$$

$$
\begin{aligned}
& \text { 5ML CdSe NPLs: } \\
& \qquad \varepsilon=10377045 \pm 3775479+1757 \pm 21(L S)^{1.60}
\end{aligned}
$$

where $L S$ is the lateral size of the NPL and must be taken here in $\mathrm{nm}^{2}$, which will give $\sigma$ in the units of $\mathrm{cm}^{-1} \mathrm{M}^{-1}$. These empiric formulations will allow for finding concentration (molarity) from the absorption measurement of the 4 and 5 ML NPL samples with known lateral size.

In addition, the molar extinction coefficient values at the energy of hh-e transition peak and at the high energy value 3.1 $\mathrm{eV}$ are listed in Table S1 (see the Supporting Information). These extinction coefficients are desirably substantially very high, which makes NPLs promising for optoelectronic applications.

To verify the results from the ICP-OES measurements, we used another spectroscopy technique, ICP-MS. The absorption cross sections for the 4 and 5 ML NPLs using the ICP-MS technique are presented in the Supporting Information. The results for the 4 ML NPLs are depicted in Figure S2a based on the $\mathrm{Se}$ concentration and Figure $\mathrm{S} 2 \mathrm{~b}$ based on the $\mathrm{Cd}$ concentration. The results for the 5 ML NPLs are also shown in Figure S3a using the Se concentration and Figure S3b using the Cd concentration. From both of the NPLs, it was observed that the absorption cross-section increases with the platelet area for the whole absorption range, and the $\sigma$ behaviors extracted using both the Se and Cd ICP-MS concentrations are consistent with those from the ICP-OES measurements. Furthermore, the trends in absorption cross-section at the transition energy of hh-e peak are strongly correlated with those observed in the ICP-OES measurements (Figures S2c and S3c).

Furthermore, we obtained the absorption cross sections per CdSe unit of the NPLs at the lowest energy absorption transition and presented the normalized ratios between the energy integrated absorption cross sections per CdSe unit in the Supporting Information of our study (Figure S4). Results for the CdSe NPLs show an approximately opposite behavior as compared to those presented for the CdSe QDs. ${ }^{35}$ We believe that these findings are also significant in terms of the comparison between the absorption behavior of the CdSe unit of QDs and NPLs. 


\section{CONCLUSIONS}

In summary, we have systematically demonstrated that the absorption cross sections and the extinction coefficients of the 4 and 5 ML NPLs strongly depend on the lateral size. The perNPL absorption cross-section and molar extinction coefficient at the hh-e energy band and the per-NPL absorption crosssection in the high photon energy regime were observed to increase substantially with the platelet area. We obtained empiric formulas relating the molar extinction coefficients to the lateral size for specific thickness ( 4 and $5 \mathrm{ML}$ ) of the NPLs, which will be very useful for calculating molarity from absorption data for the NPL samples of known lateral size. We believe these findings presented in this study will help to exploit NPLs for next-generation optoelectronic applications.

\section{ASSOCIATED CONTENT}

\section{S Supporting Information}

The Supporting Information is available free of charge on the ACS Publications website at DOI: 10.1021/acs.jpcc.5b09275.

UV-vis absorbance spectra, molar extinction coefficient values of the NPL samples, and results for the ICP-MS and also per CdSe unit (PDF)

\section{AUTHOR INFORMATION}

\section{Corresponding Author}

*E-mail: volkan@bilkent.edu.tr or hvdemir@ntu.edu.sg.

\section{Author Contributions}

The manuscript was written through contributions of all authors. All authors have given approval to the final version of the manuscript.

\section{Notes}

The authors declare no competing financial interest.

\section{ACKNOWLEDGMENTS}

The authors would like to thank the financial support from EUFP7 Nanophotonics4Energy NoE, TUBITAK EEEAG 109E002, 109E004, 110E010, 110E217, NRF-RF-2009-09, NRF-CRP-6-2010-02, and A*STAR of Singapore. H.V.D. and A.Y. acknowledge support from TUBITAK 2214A, ESFEURYI, and TUBA-GEBIP. The authors also acknowledge Kivanc Gungor for his valuable comments and members of analytical chemistry laboratory at General Directorate of Mineral Research and Exploration, TURKEY.

\section{REFERENCES}

(1) Ithurria, S.; Dubertret, B. Quasi 2D Colloidal CdSe Platelets with Thicknesses Controlled at the Atomic Level. J. Am. Chem. Soc. 2008, 130, 16504-16505.

(2) Ithurria, S.; Tessier, M. D.; Mahler, B.; Lobo, R. P. S. M.; Dubertret, B.; Efros, A. L. Colloidal Nanoplatelets with TwoDimensional Electronic Structure. Nat. Mater. 2011, 10, 936-941.

(3) Lawrence, K. N.; Johnson, M. A.; Dolai, S.; Kumbhar, A.; Sardar, R. Solvent-like Ligand-Coated Ultrasmall Cadmium Selenide Nanocrystals: Strong Electronic Coupling in a Self-Organized Assembly. Nanoscale 2015, 7, 11667-11677.

(4) Yeltik, A.; Guzelturk, B.; Hernandez-Martinez, P. L.; Govorov, A. O.; Demir, H. V. Phonon-Assisted Exciton Transfer into Silicon Using Nanoemitters: The Role of Phonons and Temperature E Ffects in Förster Resonance Energy Transfer. ACS Nano 2013, 7, 1049210501.

(5) Olutas, M.; Guzelturk, B.; Kelestemur, Y.; Yeltik, A.; Delikanli, S.; Demir, H. V. Lateral Size-Dependent Spontaneous and Stimulated
Emission Properties in Colloidal CdSe Nanoplatelets. ACS Nano 2015 9, 5041-5050.

(6) Keldysh, L. V. Coulomb Interaction in Thin Semiconductor and Semimetal Films. J. Exp Theor. Phys. Lett. 1979, 29, 658-660.

(7) Tikhodeev, S. G.; Gippius, N. A.; Yablonskii, A. L.; Dzyubenko, A. B.; Kulik, L. V.; Kulakovskii, V. D.; Forchel, A. Excitons in near Surface Quantum Wells: Local Probe of Semiconductor/vacuum Surface. Phys. Status Solidi 1997, 164, 179-182.

(8) Gippius, N. A.; Yablonskii, A. L.; Dzyubenko, A. B.; Tikhodeev, S. G.; Kulik, L. V.; Kulakovskii, V. D.; Forchel, A. Excitons in nearSurface Quantum Wells in Magnetic Fields: Experiment and Theory. J. Appl. Phys. 1998, 83, 5410-5417.

(9) Kulik, L. V.; Kulakovskii, V. D.; Bayer, M.; Forchel, A.; Gippius, N. A.; Tikhodeev, S. G. Dielectric Enhancement of Excitons in nearSurface Quantum Wells. Phys. Rev. B: Condens. Matter Mater. Phys. 1996, 54, 2335-2338.

(10) Achtstein, A. W.; Schliwa, A.; Prudnikau, A.; Hardzei, M.; Artemyev, M. V.; Thomsen, C.; Woggon, U. Electronic Structure and Exciton - Phonon Interaction in Two- Dimensional Colloidal CdSe Nanosheets. Nano Lett. 2012, 12, 3151-3157.

(11) Peter, G.; Gobel, E. O. Dependence of Radiative Exciton Lifetimes in Quantum. Phys. Rev. Lett. 1987, 59, 2337-2340.

(12) Rashba, E. I.; Gurgenishvili, G. E. Edge Absorption Theory in Semiconductors. Sov. Phys. Solid State 1962, 4, 759-760.

(13) Chen, Z.; Nadal, B.; Mahler, B.; Aubin, H.; Dubertret, B. Quasi2D Colloidal Semiconductor Nanoplatelets for Narrow Electroluminescence. Adv. Funct. Mater. 2014, 24, 295-302.

(14) Guzelturk, B.; Kelestemur, Y.; Olutas, M.; Delikanli, S.; Demir, H. V. Amplified Spontaneous Emission and Lasing in Colloidal Nanoplatelets. ACS Nano 2014, 8, 6599-6605.

(15) Grim, J. Q.; Christodoulou, S.; Di Stasio, F.; Krahne, R.; Cingolani, R.; Manna, L.; Moreels, I. Continuous-Wave Biexciton Lasing at Room Temperature Using Solution-Processed Quantum Wells. Nat. Nanotechnol. 2014, 9, 891-895.

(16) She, C.; Fedin, I.; Dolzhnikov, D. S.; Demortiére, A.; Schaller, R. D.; Pelton, M.; Talapin, D. V. Low-Threshold Stimulated Emission Using Colloidal Quantum Wells. Nano Lett. 2014, 14, 2772-2777.

(17) Yu, W. W.; Qu, L.; Guo, W.; Peng, X. Experimental Determination of the Extinction Coefficient of CdTe, CdSe, and CdS Nanocrystals. Chem. Mater. 2003, 15, 2854-2860.

(18) Tessier, M. D.; Javaux, C.; Maksimovic, I.; Loriette, V.; Dubertret, B. Spectroscopy of Single CdSe Nanoplatelets. ACS Nano 2012, 6, 6751-6758.

(19) Yeltik, A.; Kucukayan-Dogu, G.; Guzelturk, B.; Fardindoost, S.; Kelestemur, Y.; Demir, H. V. Evidence for Nonradiative Energy Transfer in Graphene Oxide Based Hybrid Structures. J. Phys. Chem. C 2013, 117, 25298-25304.

(20) Yeltik, A.; Guzelturk, B.; Ludwig Hernandez-Martinez, P.; Akhavan, S.; Demir, H. V. Excitonic Enhancement of Nonradiative Energy Transfer to Bulk Silicon with the Hybridization of Cascaded Quantum Dots. Appl. Phys. Lett. 2013, 103, 261103.

(21) Naeem, A.; Masia, F.; Christodoulou, S.; Moreels, I.; Borri, P.; Langbein, W. Evidence of Giant Oscillator Strength in the Exciton Dephasing of CdSe Nanoplatelets Measured by Resonant Four-Wave Mixing. Arxiv 2014, 1-8.

(22) Ricard, D.; Ghanassi, M.; Schanne-Klein, M. C. Dielectric Confinement and the Linear and Nonlinear Optical Properties of Semiconductor-Doped Glasses. Opt. Commun. 1994, 108, 311-318.

(23) Tessier, M. D.; Spinicelli, P.; Dupont, D.; Patriarche, G.; Ithurria, S.; Dubertret, B. Efficient Exciton Concentrators Built from Colloidal Core/Crown CdSe/CdS Semiconductor Nanoplatelets. Nano Lett. 2014, 14, 207-213.

(24) Brouwer, A. M. Standards for Photoluminescence Quantum Yield Measurements in Solution (IUPAC Technical Report). Pure Appl. Chem. 2011, 83, 2213-2228.

(25) Li, Z.; Qin, H.; Guzun, D.; Benamara, M.; Salamo, G.; Peng, X. Uniform Thickness and Colloidal-Stable CdS Quantum Disks with Tunable Thickness: Synthesis and Properties. Nano Res. 2012, 5, 337351. 
(26) Leatherdale, C. A.; Woo, W. K.; Mikulec, F. V.; Bawendi, M. G. On the Absorption Cross Section of CdSe Nanocrystal Quantum Dots. J. Phys. Chem. B 2002, 106, 7619-7622.

(27) Kuno, M. Introductory Nanoscience; Garland Science: New York, 2011.

(28) Palik, E. D. Handbook of Optical Constants of Solids; Academic Press: New York, 1998.

(29) Van de Hulst, H. C. Light Scattering by Small Particles; Dover Publications: New York, 1981.

(30) Tessier, M. D.; Javaux, C.; Maksimovic, I.; Loriette, V.; Dubertret, B. Spectroscopy of Single CdSe Nanoplatelets. ACS Nano 2012, 6, 6751-6758.

(31) Schmelz, O.; Mews, A.; Basche, T.; Herrmann, A.; Muellen, K. Supramolecular Complexes from CdSe Nanocrystals and Organic Fluorophors. Langmuir 2001, 17, 2861-2865.

(32) Striolo, A.; Ward, J.; Prausnitz, J. M.; Parak, W. J.; Zanchet, D.; Gerion, D.; Milliron, D.; Alivisatos, A. P. Molecular Weight, Osmotic Second Virial Coefficient, and Extinction Coefficient of Colloidal CdSe Nanocrystals. J. Phys. Chem. B 2002, 106, 5500-5505.

(33) Shaviv, E.; Salant, A.; Banin, U. Size Dependence of Molar Absorption Coefficients of CdSe Semiconductor Quantum Rods. ChemPhysChem 2009, 10, 1028-1031.

(34) Protasenko, V.; Bacinello, D.; Kuno, M. Experimental Determination of the Absorption Cross-Section and Molar Extinction Coefficient of CdSe and CdTe Nanowires. J. Phys. Chem. B 2006, 110, 25322-25331.

(35) de Mello Donega, C.; Koole, R. Size Dependence of the Spontaneous Emission Rate and Absorption Cross Section of CdSe and CdTe Quantum Dots. J. Phys. Chem. C 2009, 113, 6511-6520. 